PROCEEDINGS OF THE

AMERICAN MATHEMATICAL SOCIETY

Volume 137, Number 2, February 2009, Pages 579-584

S 0002-9939(08)09662-7

Article electronically published on August 19, 2008

\title{
PRODUCTS OF CESÀRO CONVERGENT SEQUENCES WITH APPLICATIONS TO CONVEX SOLID SETS AND INTEGRAL OPERATORS
}

\author{
ANTON R. SCHEP
}

(Communicated by Nigel J. Kalton)

\begin{abstract}
Let $0 \leq a_{n}, b_{n}, c_{n}$ such that $a_{n}=b_{n} c_{n}$. If $a=\lim _{n \rightarrow \infty} a_{n}$, and $\left\{b_{n}\right\}$ and $\left\{c_{n}\right\}$ Cesàro converge to $b$, respectively $c$, then $a \leq b c$. This implies that if in addition $\left\{b_{n}\right\}$ and $\left\{c_{n}\right\}$ are similarly ordered, then $a=b c$. As applications we prove that the pointwise product of two convex solid sets closed in measure is again closed in measure and a factorization result for kernels of regular integral operators on $L_{p}$-spaces.
\end{abstract}

\section{INTRODUCTION}

Recall that a sequence $\left\{a_{n}\right\}$ of real numbers is called Cesàro convergent to $a$ if $\lim _{n \rightarrow \infty} \frac{a_{1}+\cdots+a_{n}}{n}=a$. It is known that in general there is no product theorem for Cesàro convergence of sequences. In fact the product of two Cesàro convergent sequences does not have to be Cesàro convergent; e.g., if $\left\{a_{n}\right\}=$ $\{1,1,2,0, \cdots, 2,0,1,1, \cdots, 1,1,2,0,2,0, \cdots\}$, then $\left\{a_{n}\right\}$ Cesàro converges to 1 , but the averages for $a_{n}^{2}$ can be made to oscillate between 1 and 2 , by taking the consecutive blocks of 1's and 2,0's increasingly larger. In that case $\left\{a_{n}^{2}\right\}$ does not Cesàro converge. Even if we assume that the product of two Cesàro convergent sequences is again Cesàro convergent, this does not imply that there is an order relation between the Cesàro limit of the product and the product of the Cesàro limits. This is obvious when we change the sign of one of the two sequences. The main result we prove about Cesàro convergence is that if $0 \leq a_{n}, b_{n}, c_{n}$ are such that $a_{n}=b_{n} c_{n}, a=\lim _{n \rightarrow \infty} a_{n}$, and $\left\{b_{n}\right\}$ and $\left\{c_{n}\right\}$ Cesàro converge to $b$, respectively $c$, then $a \leq b c$. Recall that two sequences $\left\{b_{n}\right\}$ and $\left\{c_{n}\right\}$ are said to be similarly ordered if $\left(b_{n}-b_{m}\right)\left(c_{n}-c_{m}\right) \geq 0$ for all $n, m \geq 1$. If we have in the main result that $\left\{b_{n}\right\}$ and $\left\{c_{n}\right\}$ are also similarly ordered, then $a=b c$. To apply these results we use a theorem of Komlós for $L_{1}(X, \mu)([4)$, extended to Banach function spaces. Our first application is to the pointwise product of convex solid subsets of $L^{0}(X, \mu)$. Let $A, B$ be convex solid subsets of $L^{0}(X, \mu)$ and assume both $A$ and $B$ are closed in measure. Then we will show that the pointwise product $A \cdot B=\{f: f=g h, g \in A, h \in B\}$ is again closed in measure. This result is of interest in the case that $A$ and $B$ are unit balls of Banach function spaces with the Fatou property. Our second application involves regular integral operators on $L^{p}$.

Received by the editors January 23, 2008.

2000 Mathematics Subject Classification. Primary 40G05, 46E30, 47B34.

Key words and phrases. Cesàro convergence, convex solid sets, integral operators.

(C)2008 American Mathematical Society Reverts to public domain 28 years from publication 
By $\mathcal{K}_{r}\left(L_{p}\right)$ we shall denote the collection of all regular integral operators on $L_{p}$. If we equip $\mathcal{K}_{r}\left(L_{p}\right)$ with the regular norm $\|\cdot\|_{r}$, i.e., the operator norm of the modulus operator $|T|$, then it is well-known that $\mathcal{K}_{r}\left(L_{p}\right)$ becomes a Banach function space on $X \times X$ with the Fatou property. Our main result is here that if $T \in \mathcal{K}_{r}\left(L_{p}\right)$ with kernel $T(x, y)$, then the kernel can be written as $T(x, y)=T_{1}(x, y) T_{2}(x, y)$, where $T_{1} \in L_{\infty, p^{\prime}}$ and $T_{2} \in L_{\infty, p}^{t}$. Moreover $T_{1}$ and $T_{2}$ can be chosen such that $\|T\|_{r}=\left\|T_{1}\right\|_{\infty, p^{\prime}}\left\|T_{2}^{t}\right\|_{\infty, p}$.

\section{A product theorem for Cesìro CONVERGEnCE}

We start with two lemmas.

Lemma 2.1. Let $0<a_{k} \in \mathbb{R}$ for $1 \leq k \leq n$. Then

$$
1 \leq\left(\frac{1}{n} \sum_{k=1}^{n} a_{k}\right)\left(\frac{1}{n} \sum_{k=1}^{n} \frac{1}{a_{k}}\right) \text {. }
$$

Proof. From the Arithmetic-Geometric Mean inequality we have

$$
\frac{1}{n} \sum_{k=1}^{n} a_{k} \geq\left(a_{1} \cdots a_{n}\right)^{\frac{1}{n}}
$$

and

$$
\frac{1}{n} \sum_{k=1}^{n} \frac{1}{a_{k}} \geq\left(\frac{1}{a_{1}} \cdots \frac{1}{a_{n}}\right)^{\frac{1}{n}}
$$

Taking products on the left and right now yields the desired inequality.

Lemma 2.2. Let $0<a_{n}, b_{n} \in \mathbb{R}$ for $n \in \mathbb{N}$ such that $\lim _{n \rightarrow \infty} a_{n}=a>0$ and $\lim _{n \rightarrow \infty} \frac{1}{n}\left(b_{1}+\cdots+b_{n}\right)=b$. Then

$$
\lim _{n \rightarrow \infty} \frac{a_{n}}{n}\left(\frac{b_{1}}{a_{1}}+\cdots+\frac{b_{n}}{a_{n}}\right)=b .
$$

Proof. Let $\epsilon>0$ and let $\delta>0$ such that $a_{n}>\delta$ for all $n \geq 1$. Then there exists $N>1$ such that $\left|a_{n}-a_{m}\right|<\delta \epsilon$ for all $n, m \geq N$. This implies that

$$
\left|\frac{a_{n}}{a_{m}}-1\right|<\frac{\delta \epsilon}{a_{m}}<\epsilon
$$

for all $n, m \geq N$. Hence it follows that

$$
\begin{aligned}
\left|\frac{a_{n}}{n}\left(\frac{b_{1}}{a_{1}}+\cdots+\frac{b_{n}}{a_{n}}\right)-\frac{1}{n}\left(b_{1}+\cdots+b_{n}\right)\right| & =\frac{1}{n}\left|\left(\frac{a_{n}}{a_{1}}-1\right) b_{1}+\cdots+\left(\frac{a_{n}}{a_{n}}-1\right) b_{n}\right| \\
& \leq \frac{1}{n} \sum_{k=1}^{N-1}\left|\frac{a_{n}}{a_{k}}-1\right|\left|b_{k}\right|+\frac{\epsilon}{n} \sum_{k=N}^{n-1} b_{k}
\end{aligned}
$$

for all $n \geq N$. This implies that

$$
\limsup _{n}\left|\frac{a_{n}}{n}\left(\frac{b_{1}}{a_{1}}+\cdots+\frac{b_{n}}{a_{n}}\right)-\frac{1}{n}\left(b_{1}+\cdots+b_{n}\right)\right| \leq \epsilon b
$$

for all $\epsilon>0$, from which the result follows.

Theorem 2.3. Let $0<a_{n}, b_{n}, c_{n} \in \mathbb{R}$ for $n \in \mathbb{N}$ with $a_{n}=b_{n} c_{n}$ such that $\lim _{n \rightarrow \infty} a_{n}=a, \lim _{n \rightarrow \infty} \frac{1}{n}\left(b_{1}+\cdots+b_{n}\right)=b$ and $\lim _{n \rightarrow \infty} \frac{1}{n}\left(c_{1}+\cdots+c_{n}\right)=c$. Then $a \leq b c$. In particular $b \neq 0$ and $c \neq 0$, whenever $a>0$. 
Proof. If $a=0$, then there is nothing to prove. Assume therefore that $a>0$. Let $\tilde{b}_{n}=\frac{1}{n}\left(b_{1}+\cdots+b_{n}\right)$ and put $\tilde{c}_{n}=\frac{a_{n}}{b_{n}}$. Then $\lim _{n \rightarrow \infty} \tilde{c}_{n}=\frac{a}{b}$ if $b \neq 0$ and $\lim _{n \rightarrow \infty} \tilde{c}_{n}=\infty$ if $b=0$. From Lemmas 2.1 and 2.2 we have

$$
\tilde{c}_{n} \leq \frac{a_{n}}{n}\left(\frac{1}{b_{1}}+\cdots+\frac{1}{b_{n}}\right)=\frac{a_{n}}{n}\left(\frac{c_{1}}{a_{1}}+\cdots+\frac{c_{n}}{a_{n}}\right) \rightarrow c .
$$

This shows that $\lim _{n \rightarrow \infty} \tilde{c}_{n} \leq c<\infty$, so $b \neq 0$ and $\frac{a}{b}=\lim _{n \rightarrow \infty} \tilde{c}_{n} \leq c$.

Remark 2.4. The above theorem is no longer true if we replace the condition that $\left\{a_{n}\right\}$ is convergent by the condition that $\left\{a_{n}\right\}$ is Cesàro convergent. To see this take $\left\{a_{n}\right\}=\{4,1,4,1, \cdots\}$ and $\left\{b_{n}\right\}=\left\{c_{n}\right\}=\{2,1,2,1, \cdots\}$. Then $\left\{a_{n}\right\}$ is Cesàro convergent to $\frac{5}{2}$, and $\left\{b_{n}\right\}$ and $\left\{c_{n}\right\}$ are Cesàro convergent to $\frac{3}{2}$. However $\left(\frac{3}{2}\right)^{2}=\frac{9}{4}<\frac{5}{2}$.

To get equality in the above theorem, we recall first the following terminology. Two sequences $\left\{b_{n}\right\}$ and $\left\{c_{n}\right\}$ are said to be similarly ordered if

$$
\left(b_{n}-b_{m}\right)\left(c_{n}-c_{m}\right) \geq 0
$$

for all $n, m \geq 1$. The following inequality is called Tchebychef's inequality in [2](Item 43).

Lemma 2.5. If $\left\{b_{n}\right\}$ and $\left\{c_{n}\right\}$ are similarly ordered, then for all $n \geq 1$ we have

$$
\left(\frac{1}{n} \sum_{k=1}^{n} b_{k}\right)\left(\frac{1}{n} \sum_{k=1}^{n} c_{k}\right) \leq\left(\frac{1}{n} \sum_{k=1}^{n} b_{k} c_{k}\right) .
$$

Combining this lemma with Theorem 2.3 we get immediately the following theorem.

Theorem 2.6. Let $0<a_{n}, b_{n}, c_{n} \in \mathbb{R}$ for $n \in \mathbb{N}$ with $a_{n}=b_{n} c_{n}$ such that $\lim _{n \rightarrow \infty} a_{n}=a, \lim _{n \rightarrow \infty} \frac{1}{n}\left(b_{1}+\cdots+b_{n}\right)=b$ and $\lim _{n \rightarrow \infty} \frac{1}{n}\left(c_{1}+\cdots c_{n}\right)=c$. Assume $\left\{b_{n}\right\}$ and $\left\{c_{n}\right\}$ are similarly ordered. Then $a=b c$.

\section{An application to the Pointwise PRoduct of CONVEX SOlid SETS}

Let $(X, \Sigma, \mu)$ be a complete finite measure space. By $L_{0}(X, \mu)$ we will denote the set of all measurable functions which are finite a.e. As usual we will identify functions equal almost everywhere. An ideal $E$ of $L_{0}(X, \mu)$ equipped with a lattice norm is called a Köthe function space; i.e., if $f \in E$ and $|g| \leq|f|$ a.e., then $g \in E$ and $\|g\| \leq\|f\|$. A norm complete Köthe function space is called a Banach function space. For a detailed treatment of Banach function spaces we refer to [9]. The detailed study of Banach function spaces led to the study of Riesz spaces and Banach lattices, which incorporated, clarified and extended the earlier theory; see e.g. [10].

To apply the above results we will use the following theorem, which is a direct consequence of a fundamental theorem of Komlós for $L_{1}(X, \mu)$ [4].

Theorem 3.1. Let $E$ be a normed Köthe space and $\left\{f_{n}\right\}$ a norm bounded sequence in $E$. Then there exist a subsequence $\left\{f_{n_{k}}\right\}$ of $\left\{f_{n}\right\}$ and $f \in E^{\prime \prime}$ such that $\left\{f_{n_{k}}\right\}$ and any subsequence of $\left\{f_{n_{k}}\right\}$ Cesàro converges to $f$ a.e. on $X$. 
Proof. Let $0<g \in E^{\prime}$ be strictly positive so that $\mu$ and $g d \mu$ are equivalent measures. Then $\left\{f_{n}\right\}$ is a norm bounded sequence in $L_{1}(X, g d \mu)$ and the existence of $0 \leq f \in$ $L_{1}(X, g d \mu)$ and a subsequence with the stated a.e. convergence properties follows then from Komlós' theorem [4. To see that $f \in E^{\prime \prime}$, note that $\left\|f_{n}\right\|_{E} \leq C$ for all $n$ implies that also $\left\|f_{n}\right\|_{E^{\prime \prime}} \leq C$. Now $\left\|\frac{1}{k}\left(f_{n_{1}}+\cdots+f_{n_{k}}\right)\right\|_{E^{\prime \prime}} \leq C$ implies by the Fatou property of $\|\cdot\|_{E^{\prime \prime}}$ that also $\|f\|_{E^{\prime \prime}} \leq C$.

Theorem 3.2. Let $A$ and $B$ be convex solid subsets of $L_{0}(X, \mu)$, which are closed in measure. Then $A \cdot B$ is closed in measure.

Proof. Assume first that at least one of $A$ and $B$ is not bounded in measure; say $A$ is not bounded in measure. Then by Theorem 11 of [6] there exists a measurable set $X_{0}$ such that $A_{\mid X_{0}}=L_{0}\left(X_{0}, \mu\right)$ and $A_{\mid X_{0}^{c}}$ is bounded in measure. This implies then that $A_{\mid X_{0}} \cdot B=L_{0}\left(X_{1}, \mu\right)$ for some measurable subset of $X_{0}$. In particular $A_{\mid X_{0}} \cdot B$ is closed in measure. Assume therefore that both $A$ and $B$ are bounded in measure. By the same theorem of [6] we get that $A$ and $B$ are unit balls of Banach function spaces $E$, respectively $F$, with the Fatou property. Let $0 \leq f_{n} \in A \cdot B$ be such that $f_{n}(x) \rightarrow f(x)$ a.e., where $f \in L_{0}(X, \mu)$. Then $f_{n}=g_{n} h_{n}$, where $\left\|g_{n}\right\|_{E} \leq 1$ and $\left\|h_{n}\right\|_{F} \leq 1$ for all $n \geq 1$. By Komlós' theorem there exist subsequences $\left\{g_{n_{k}}\right\}$ and $\left\{h_{n_{k}}\right\}$ such that $\left\{g_{n_{k}}\right\}$ Cesàro converges a.e. to $g \in E$ with $\|g\|_{E} \leq 1$ and $\left\{h_{n_{k}}\right\}$ Cesàro converges a.e. to $h \in F$ with $\|h\|_{F} \leq 1$. By the above theorem on Cesàro convergence we get that $f \leq g h$ a.e. Thus $f \in A \cdot B$ and the proof is complete.

\section{AN APPLiCATION TO INTEGRAL OPERATORS ON $L_{p}$-SPACES}

We recall the definition of regular integral or kernel operators on $L_{p}$-spaces. Let $T(x, y)$ be a $\mu \times \mu$-measurable function on $X \times X$. Then $T(x, y)$ is the kernel of an integral operator $T$ from $L_{p}$ into $L_{p}$ if

$$
\int_{X}|T(x, y) f(y)| d \mu(y)<\infty \text { a.e. }
$$

for all $f \in L_{p}$ and

$$
T f(x)=\int_{X} T(x, y) f(y) d \mu(y) \in L_{p}
$$

for all $f \in L_{p}$. If in addition $|T(x, y)|$ is the kernel of an integral operator (denoted by $|T|$ ) from $L_{p}$ into $L_{p}$, then $T$ is called a regular (or order bounded) integral operator. By $\mathcal{K}_{r}\left(L_{p}\right)$ we shall denote the collection of all such regular integral operators on $L_{p}$. If we equip $\mathcal{K}_{r}\left(L_{p}\right)$ with the regular norm $\|\cdot\|_{r}$, i.e., the operator norm of the modulus operator $|T|$, then it is well-known that $\mathcal{K}_{r}\left(L_{p}\right)$ becomes a Banach function space on $X \times X$ with the Fatou property. Many order-theoretic properties of $\mathcal{K}_{r}\left(L_{p}\right)$ are known (see e.g. [10]), but there does not exists an explicit formula for $\|T\|_{r}$ in terms of its kernel $T(x, y)$ in case $1<p<\infty$, even not for the matrix case. Therefore it might be of some interest to prove that every $T \in \mathcal{K}_{r}\left(L_{p}\right)$ is in fact a product of two kernels in explicitly defined Banach function spaces. For a measurable function $F$ on $X \times X$ we define for $1 \leq p<\infty$ the norm $\|F\|_{\infty, p}$ as follows:

We write

$$
\|F\|_{\infty, p}=\left\|\left(\int|F(x, y)|^{p} d \mu(y)\right)^{\frac{1}{p}}\right\|_{\infty} .
$$

$$
L_{\infty, p}=\left\{F \in L_{0}(X \times X):\|F\|_{\infty, p}<\infty\right\} .
$$


One can show that $L_{\infty, p}$ is a Banach function space with the Fatou property, isometric to the collection of all bounded operators from $L_{p^{\prime}}$ into $L_{\infty}$ provided with the operator norm. Given $F$ on $X \times X$ we define the transpose of $F$ by $F^{t}(x, y)=F(y, x)$. Then $L_{\infty, p}^{t}$ will denote the collection of all $F$ such that $F^{t} \in$ $L_{\infty, p}$ and the norm on $L_{\infty, p}^{t}$ will be defined by $\left\|F^{t}\right\|_{\infty, p}$. The Banach function space $L_{\infty, p}^{t}$ is for $1<p \leq \infty$ isometric with the collection of all bounded operators from $L_{1}$ into $L_{p}$ with the operator norm.

Theorem 4.1. Let $1<p<\infty$ and $0 \leq T(x, y) \in L_{0}(X \times X)$. Then $T \in \mathcal{K}_{r}\left(L_{p}\right)$ if and only if the kernel $T(x, y)$ can be written as $T(x, y)=T_{1}(x, y) T_{2}(x, y)$, where $T_{1} \in L_{\infty, p^{\prime}}$ and $T_{2} \in L_{\infty, p}^{t}$. Moreover if $T \in \mathcal{K}_{r}\left(L_{p}\right)$, then $T_{1}$ and $T_{2}$ can be chosen such that $\|T\|_{r}=\left\|T_{1}\right\|_{\infty, p^{\prime}}\left\|T_{2}^{t}\right\|_{\infty, p}$.

Proof. Assume first that $T_{1} \in L_{\infty, p^{\prime}}$ and $T_{2}^{t} \in L_{\infty, p}$ and let $T(x, y)=$ $T_{1}(x, y) T_{2}(x, y)$. Denote by $T$ the integral operator with kernel $T(x, y)$. Then, for $f \in L_{p}$, we have

$$
\begin{aligned}
|T f(x)| & \leq \int\left|T_{1}(x, y)\right|\left|T_{2}(x, y)\right||f(y)| d \mu(y) \\
& \leq\left(\int\left|T_{1}(x, y)\right|^{p^{\prime}} d \mu(y)\right)^{\frac{1}{p^{\prime}}}\left(\int\left|T_{2}(x, y)\right|^{p}|f(y)|^{p} d \mu(y)\right)^{\frac{1}{p}} \\
& \leq\left\|T_{1}\right\|_{\infty, p^{\prime}}\left(\int\left|T_{2}(x, y)\right|^{p}|f(y)|^{p} d \mu(y)\right)^{\frac{1}{p}} .
\end{aligned}
$$

Hence

$$
\begin{aligned}
\int|T f(x)|^{p} d \mu(x) & \leq\left\|T_{1}\right\|_{\infty, p^{\prime}}^{p} \int\left(\int\left|T_{2}(x, y)\right|^{p}|f(y)|^{p} d \mu(y)\right) d \mu(x) \\
& =\left\|T_{1}\right\|_{\infty, p^{\prime}}^{p} \int\left(\int\left|T_{2}(x, y)\right|^{p} d \mu(x)\right)|f(y)|^{p} d \mu(y) \\
& \leq\left\|T_{1}\right\|_{\infty, p^{\prime}}^{p}\left\|T_{2}^{t}\right\|_{\infty, p}^{p}\|f\|_{p}^{p} .
\end{aligned}
$$

It follows that $T \in \mathcal{K}_{r}\left(L_{p}\right)$ and $\|T\|_{r} \leq\left\|T_{1}\right\|_{\infty, p^{\prime}}\left\|T_{2}^{t}\right\|_{\infty, p}$. Now let $T \in \mathcal{K}_{r}\left(L_{p}\right)$. Then we can assume that $0 \leq T$ and $\|T\|=1$. Let $\epsilon>0$. Then by Gagliardo's converse of the Schur test for positive linear operators (see e.g. [3]) there exists $0<$ $f_{0} \in L_{p}$ with $\left\|f_{0}\right\|_{p}=1$ such that $T^{*}\left(T f_{0}\right)^{p-1} \leq(1+\epsilon) f_{0}^{p-1}$. Now define $T_{1}(x, y)=$ $T(x, y)^{\frac{1}{p^{\prime}}} f_{0}(y)^{\frac{1}{p^{\prime}}}\left(T f_{0}(x)\right)^{-\frac{1}{p^{\prime}}}$ and $T_{2}(x, y)=T(x, y)^{\frac{1}{p}} f_{0}(y)^{-\frac{1}{p^{\prime}}}\left(T f_{0}(x)\right)^{\frac{1}{p^{\prime}}}$. Then clearly $T(x, y)=T_{1}(x, y) T_{2}(x, y)$. Moreover

$$
\int T_{1}(x, y)^{p^{\prime}} d \mu(y)=T f_{0}(x)\left(T f_{0}(x)\right)^{-1}=1 \text { a.e. }
$$

and

$$
\int T_{2}(x, y)^{p} d \mu(x)=T^{*}\left(T f_{0}\right)^{p-1}(y) \cdot f_{0}(y)^{1-p} \leq 1+\epsilon \text { a.e. }
$$

This shows that $T_{1} \in L_{\infty, p^{\prime}}$ and $T_{2}^{t} \in L_{\infty, p}$ and $\left\|T_{1}\right\|_{\infty, p^{\prime}}\left\|T_{2}^{t}\right\|_{\infty, p} \leq 1+\epsilon$. Hence $\|T\|_{r}=\inf \left\{\left\|T_{1}\right\|_{\infty, p^{\prime}}\left\|T_{2}^{t}\right\|_{\infty, p}:|T(x, y)|=\left|T_{1}(x, y) T_{2}(x, y)\right|, T_{1} \in L_{\infty, p^{\prime}}, T_{2}^{t} \in\right.$ $\left.L_{\infty, p}\right\}$. We will now show that the infimum is actually a minimum. Again let $T \in \mathcal{K}_{r}\left(L_{p}\right)$ with $\|T\|_{r}=1$. Then we can find $0 \leq T_{1, n} \in L_{\infty, p^{\prime}}$ and $0 \leq$ $T_{2, n}^{t} \in L_{\infty, p}$ such that $|T(x, y)|=T_{1, n}(x, y) T_{2, n}(x, y)$ and $\left\|T_{1, n}\right\|_{\infty, p^{\prime}}=1$ and $\left\|T_{2, n}^{t}\right\|_{\infty, p} \leq 1+\frac{1}{2^{n}}$. From Komlós' theorem it follows that there exist subsequences 
$\left\{T_{1, n_{k}}\right\}$ and $\left\{T_{2, n_{k}}\right\}, T_{1} \in L_{\infty, p^{\prime}}$ and $T_{2} \in L_{\infty, p}^{t}$ such that $\left\{T_{1, n_{k}}\right\}$ Cesàro converges a.e. to $T_{1}$ and $\left\{T_{2, n_{k}}\right\}$ Cesàro converges a.e. to $T_{2}$. From Theorem 2.3 it follows that $|T| \leq T_{1} T_{2}$ a.e. By replacing $T_{2}$ by the smaller function $|T| / T_{1}$, on the set where $T_{2} \neq 0$, we can assume that $|T|=T_{1} T_{2}$. Clearly $\left\|T_{1}\right\|_{\infty, p} \leq 1$ and

$$
\left\|\frac{1}{k}\left(T_{2, n_{1}}+\cdots+T_{2, n_{k}}\right)\right\|_{L_{\infty, p}^{t}} \leq \frac{1}{k}\left(1+\frac{1}{2^{n_{1}}}+\cdots+1+\frac{1}{2^{n_{k}}}\right) \leq 1+\frac{1}{k}
$$

implies that $\left\|T_{2}^{t}\right\|_{\infty, p} \leq 1$. This implies that both $\left\|T_{1}\right\|_{\infty, p}=1$ and $\left\|T_{2}^{t}\right\|_{\infty, p}=1$. Now replacing $T_{1}$ by $\operatorname{sgn}(T) T_{1}$, we get that $T=T_{1} T_{2}$, where $T_{1}, T_{2}$ satisfy the same norm conditions as before.

Remark 4.2. One can rewrite the above factorization as $\mathcal{K}_{r}\left(L_{p}\right)=\left(L_{\infty, 1}\right)^{\frac{1}{p^{\prime}}}\left(L_{\infty, 1}^{t}\right)^{\frac{1}{p}}$. In this form the above theorem is a specialization of Pisier's result [5] that $\mathcal{L}_{r}\left(L_{p}\right)=$ $\mathcal{L}\left(L_{\infty}\right)^{\frac{1}{p^{\prime}}} \mathcal{L}\left(L_{1}\right)^{\frac{1}{p}}$. Pisier's result was already anticipated for positive operators by Akcoglu, Baxter and Lee in [1]. We also note that for $p=2$ the implication $T=T_{1} T_{2}$, where $T_{1} \in L_{\infty, 2}$ and $T_{2} \in L_{\infty, 2}^{t}$, implies that $T$ is bounded on $L_{2}$ with norm less than or equal to $\left\|T_{1}\right\|_{\infty, 2}\left\|T_{2}^{t}\right\|_{\infty, 2}$ (see Theorem 6.34 of [8]). The above theorem shows that an exact converse is true, i.e., that $\|T\|_{r}=\left\|T_{1}\right\|_{\infty, p^{\prime}}\left\|T_{2}^{t}\right\|_{\infty, p}$ in all of these results. This is somewhat unexpected, as the theorem and its proof are very closely related to the Schur criterion and its converse. For the converse of the Schur criterion as used in the above proof it is known that we cannot put $\epsilon=0$ (see [7] for a discussion of this and a related open problem).

\section{REFERENCES}

1. M. A. Akcoglu, J. R. Baxter, and W. M. F. Lee, Representation of positive operators and alternating sequences, Adv. Math. 87 (1991), no. 2, 249-290. MR1112627(92i:47006)

2. G. H. Hardy, J. E. Littlewood, and G. Pólya, Inequalities, Cambridge Mathematical Library, Cambridge University Press, Cambridge, 1988, reprint of the 1952 edition. MR.944909 (89d:26016)

3. Ralph Howard and Anton R. Schep, Norms of positive operators on $L^{p}$-spaces, Proc. Amer. Math. Soc. 109 (1990), no. 1, 135-146. MR1000156 (90j:47031)

4. J. Komlós, A generalization of a problem of Steinhaus, Acta Math. Acad. Sci. Hungar. 18 (1967), 217-229. MR0210177(35:1071)

5. Gilles Pisier, Complex interpolation and regular operators between Banach lattices, Arch. Math. (Basel) 62 (1994), no. 3, 261-269. MR1259842 (95a:46027)

6. Anton R. Schep, Convex solid subsets of $L_{0}(X, \mu)$, Positivity 9 (2005), no. 3, 491-499. MR $2188533(2007 \mathrm{~m}: 46042)$

7. Anton R. Schep, Positive operators on $L^{p}$-spaces, Positivity (A. Triki, K. Boulabar, and G. Buskes, eds.), Trends in Mathematics, Birkhäuser, Basel, 2007, pp. 229-254. MR2382220

8. Joachim Weidmann, Linear operators in Hilbert spaces, Graduate Texts in Mathematics, vol. 68, Springer-Verlag, New York, 1980. MR566954 (81e:47001)

9. A. C. Zaanen, Integration, Completely revised edition of An introduction to the theory of integration, North-Holland Publishing Co., Amsterdam, 1967. MR0222234 (36:5286)

10. _ Riesz spaces. II, North-Holland Mathematical Library, vol. 30, North-Holland Publishing Co., Amsterdam, 1983. MR704021 (86b:46001)

Department of Mathematics, University of South Carolina, Columbia, South CaroLINA 29208

E-mail address: schep@math.sc.edu 